\title{
Importance of lipid ratios for predicting intracranial atherosclerotic stenosis
}

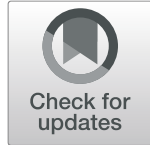

Wen-Song Yang ${ }^{1,2+}$, Rui Li ${ }^{3 \dagger}$, Yi-Qing Shen ${ }^{1,2+}$, Xing-Chen Wang ${ }^{1,4}$, Qing-Jun Liư ${ }^{5}$, Hai-Yang Wang ${ }^{2}$, Qi Li ${ }^{1,2^{*}}$, Guo-En $Y_{a o^{6 *}}$ and Peng Xie ${ }^{1,2^{*}}$

\begin{abstract}
Background: This study aims to investigate the association of lipid ratios with intracranial atherosclerotic stenosis (ICAS) in a Chinese population.

Methods: This cross-sectional study included 658 consecutive patients with ischemic stroke. Intracranial and extracranial arteries were evaluated for atherosclerotic stenosis using digital subtraction angiography or computed tomography angiography. Lipid ratios [total cholesterol (TC)/high-density lipoprotein-cholesterol (HDL-C), triglycerides (TG)/HDL-C, lowdensity lipoprotein-cholesterol (LDL-C)/HDL-C, non-high-density lipoprotein-cholesterol (non-HDL-C)/HDL-C, remnant cholesterol (RC)/HDL-C, apolipoprotein B (apo B)/apolipoprotein A-I (apo A-l), and apo B/HDL-C] were calculated.

Results: The TC/HDL-C, LDL-C/HDL-C, RC/HDL-C, non-HDL-C/HDL-C, apo B/HDL-C and apo B/apo A-I ratios (all $P<0.05$ ) were significantly associated with ICAS but not with extracranial atherosclerotic stenosis after adjustment for confounding factors. Receiver operating characteristic (ROC) curves analysis revealed that the apo B/apo A-I ratio had the largest area under the ROC curve (AUC) among lipid levels alone and for lipid ratios ( $A \cup C=0.588$ ). Lipid ratios had higher AUC values than those for lipid levels alone for the identification of ICAS.

Conclusion: The TC/HDL-C, LDL-C/HDL-C, RC/HDL-C, non-HDL-C/HDL-C apo B/HDL-C, and apo B/apo A-I ratios were significantly related to ICAS risk. Compared with the other variables tested, the apo B/apo A-I ratio appeared to be a better discriminator for identifying ICAS risk in stroke patients.
\end{abstract}

Keywords: Apo B/apo A-I, Intracranial atherosclerotic stenosis, Lipid ratios, Remnant cholesterol, Stroke, Atherosclerosis, Symptomatic

\section{Take home message}

Lipid ratios could be more valuable than lipid levels alone for predicting ICAS risk, and sex-based differences were examined regarding the predictive accuracy of lipid profiles.

\footnotetext{
*Correspondence: qili_md@126.com; yaoguoensdy@sina.com; peng_xie@yahoo.com

${ }^{+}$Wen-Song Yang, Rui Li and Yi-Qing Shen contributed equally to this work. 'Department of Neurology, The First Affiliated Hospital of Chongqing Medical University, Chongqing 400016, China

${ }^{6}$ Department of Neurology, First Affiliated Hospital, PLA General Hospital, Beijing 100048, China

Full list of author information is available at the end of the article
}

\section{Background}

Intracranial atherosclerotic stenosis (ICAS) is a major cause of ischemic stroke worldwide [1,2]. The prevalence of ICAS is relatively high in Asians, whereas extracranial atherosclerotic stenosis (ECAS) is more common in Caucasians. The exact causes for this distribution of cerebral atherosclerosis are not clear, but racial differences, socioeconomic status, and risk factors may contribute to this phenomenon [3]. Because of the detrimental effects of ICAS, a better understanding of its risk factors is particularly important.

The role of lipid parameters and lipid ratios in ICAS is controversial. Low-density lipoprotein cholesterol 
(LDL-C) is associated mainly with ECAS [4]. In Chinese populations, high-density lipoprotein-cholesterol (HDL-C) [5], non-high-density lipoprotein-cholesterol (non-HDLC) [6] and total cholesterol (TC) [7] levels are associated with an increased risk of ICAS. One study based on a Korean population revealed the importance of hypercholesterolemia in ICAS in men [8]. Concerning lipid ratios, Park and colleagues indicated the importance of the apolipoprotein B/apolipoprotein A-I (apoB/apo A-I) ratio for ICAS [9]. Levels of HDL-C along with those of remnant cholesterol (RC) and a low apo B/apo A-I ratio are related to the prevention of the angiographic progression of ICAS [10]. Recently, a study found that acute ischemic stroke patients with ICAS have a higher ApoB/AI ratio was meaningfully higher than those without ICAS in both stroke group and non-stroke groups [11]. The LDL-C/ HDL-C ratio is regarded as a marker for carotid intima-media thickness (CIMT) progression [12]. In addition, several lipid ratios (apo B/apo A-I, TC/HDLC, LDL-C/HDL-C, and non-HDL-C/HDL-C) have shown clinically important correlations with coronary artery lesions, insulin resistance, and metabolic syndrome [13-15]. Moreover, higher LDL-C and lower HDL-C were associated with the presence of ICAS in a population-based study [16].

Lipid ratios are considered to be good parameters for identifying vascular diseases. Nevertheless, studies focusing on the association between lipid ratios and cerebral atherosclerosis are lacking. Additionally, the potential predictive importance of these lipid ratios in Chinese populations remains unclear. It is important to screen relevant applicable lipid ratios to identify high-risk populations. Therefore, this study compared the correlation between lipid ratios (TC/HDL-C, triglycerides (TG)/ HDL-C, LDL-C/HDL-C, RC/HDL-C, non-HDL-C/HDL$\mathrm{C}$, apo B/HDL-C, and apo B/apo A-I) and the risk of cerebral atherosclerosis in patients with ischemic stroke.

\section{Methods}

\section{Population and procedures}

The study protocol was approved by the ethics committee of the First Affiliated Hospital of Chongqing Medical University (Chongqing, China). Written informed consent was obtained from all patients.

From November 2015 to January 2017, consecutive patients with transient ischemic attack or acute $(<7$ days after onset) ischemic stroke admitted to the division within the First Affiliated Hospital of Chongqing Medical University were recruited prospectively into the study. All patients underwent cerebral digital subtraction angiography (DSA) or computed tomography angiography (CTA) to check for atherosclerotic lesions.

The exclusion criteria consisted of patients with contraindications to or who refused to undergo CTA or
DSA; non-atherosclerotic arterial stenosis or totally occlusive stenosis in intracranial and extracranial vessels; and incomplete clinical information.

\section{Collection of clinical information}

Information of each patient was acquired by completing a detailed questionnaire combined with a standardized interview. Information was obtained regarding demographics, hypertension, diabetes mellitus (DM), previous stroke, coronary heart disease, medical history, and current smoking. Blood pressure was determined with mercury manometers while the patient was seated after 10 min of rest. The parameter of "current smoking" was self-reported by patients who had smoked more than 100 cigarettes and smoked every day or some days now. The diagnoses of hypertension, DM, coronary heart disease, and previous stroke were determined according to those detailed in the International Classification of Diseases (9th revision).

\section{Laboratory measurements}

Fasting blood samples were extracted and analyzed in the laboratory department of the First Affiliated Hospital. Lipid levels were measured using a fully automatic biochemistry analyzer (Cobas c701; Roche, Basel, Switzerland) and original reagents in the laboratory department. TC, TG, HDL$\mathrm{C}$, and LDL-C levels were measured using an enzymology assay. Apo A-I and apo B levels were measured by an immunoturbidimetric assay. The non-HDL-C level was defined as the TC level minus the HDL-C level [17]. The RC level was defined as the non-HDL-C level minus the LDL$C$ level [18]. Additionally, the TC/HDL-C, TG/HDL-C, LDL-C/HDL-C, RC/HDL-C, non-HDL-C/HDL-C, apo B/ HDL-C, and apo B/apo A-I lipid ratios were calculated in this study.

\section{Assessment of cerebral atherosclerotic stenosis}

ICAS was evaluated with the method used in the Warfarin-Aspirin Symptomatic Intracranial Disease Trial [19]. ECAS was evaluated based on the method used in the North American Symptomatic Carotid Endarterectomy Trial [20]. Intracranial vessels included the anterior, middle, and posterior cerebral arteries, the basilar artery, and intracranial portions of the vertebral and internal carotid artery. Extracranial vessels included extracranial segments of the vertebral artery and internal carotid artery. The presence of ICAS or ECAS was defined as stenosis $\geq 50 \%$ in large intracranial or extracranial vessels.

\section{Statistical analyses}

Data were analyzed using SPSS 18.0 (Chicago, IL, USA) and MedCalc 11.4.2.0 (MedCalc, Ostend, Belgium). General characteristics were compared separately among 
patients between men and women, or among the groups of no cerebral atherosclerotic stenosis (NCAS), isolated ICAS, isolated ECAS, and ICAS combined ECAS. The frequency data were analyzed using the Student's t-test or one-way analysis of variance (ANOVA) if the data obey the normal distribution and the variance is homogeneous. Mann-Whitney $U$-test was performed if the frequency data did not obey the normal distribution. The categorical variables were analyzed using the chi-square test. The results are expressed as the mean \pm standard deviation for normal data or median and interquartile range for skewed data. Multiple logistic regression was carried out to evaluate the correlation of lipid ratios with ICAS and ECAS. Crude and adjusted odds ratios (ORs) with 95\% confidence intervals (CIs) were obtained. Receiver operating characteristic (ROC) curves were created to assess whether variables could predict ICAS, and the results are expressed as the area under the curve (AUC). AUC values were compared using the Z-statistic. A value of $P<0.05$ was considered significant.

\section{Results}

Six hundred and fifty-eight patients (441 men and 217 women, mean age, $65.9 \pm 11.2$ years) with transient ischemic attack or acute ischemic stroke were included in the final analysis. Of the 658 patients analyzed, DSA was performed in 24 patients (3.6\%) and the remaining 634 patients underwent CTA. The patients were divided into four subgroups as follows: No cerebral atherosclerotic stenosis (NCAS) (55.6\%), Isolated ICAS (27.7\%), Isolated ECAS (7.0\%), and ICAS combined with ECAS (9.7\%). The characteristics of the study participants in the groups of NCAS, isolated ICAS, isolated ECAS, and ICAS combined with ECAS groups were shown in Supplementary Table 1.

Sex-specific characteristics were shown in Table 1. Women were older and had lower diastolic blood pressure, and non-HDL-C/HDL-C, TC/HDL-C, RC/HDL-C, LDL-C/HDL-C, apo B/HDL-C, and apo B/apo A-I ratios than men. A significantly higher proportion of current smokers were men $(P<0.001)$, and their TC, HDL-C, and apo A-I levels were lower than those in women. Multivariable analysis of ICAS and (or) ECAS according to gender was shown in Supplementary Table 2.

Each lipid ratio was examined based on sex to assess its relationship with the risk of ICAS or ECAS. In this study, the lipid ratio in the fourth quartile was compared with that of the first quartile. The corresponding OR and $95 \% \mathrm{CI}$ of lipid ratios (TC/HDL-C, LDL-C/HDL-C, TG/HDL-C, RC/HDL-C, non-HDL-C/HDL-C, apo B/ apo A-I, and apo B/HDL-C) in the first quartile are listed (see Fig. 1). Compared with other lipid ratios, apo B/apo A-I showed the strongest relationship with ICAS after adjustment for potential confounding factors (first quartile vs. fourth quartile; [ $\beta$ ] 0.84, [OR], 2.32; [95\% CI], 1.44-3.73). Moreover, after adjustment for age, sex, current smoking, hypertension, DM, previous stroke, and coronary heart disease, this significant relationship was also observed for other lipid ratios (TC/HDL-C, $[\beta]$ 0.71, [OR] 2.04, [95\% CI] 1.26-3.31; RC/HDL-C, 0.49, $1.64,1.01-2.65$; non-HDL-C/HDL-C, 0.71, 2.04, 1.263.31; LDL-C/HDL-C, 0.65, 1.92, 1.19-3.10; apo B/HDLC, 0.83, 2.28, 1.41-3.71; fourth quartile vs. first quartile; see Fig. 1a and Supplementary Table 3). These adjusted logistic regression analyses were repeated for ECAS, but no significant association was observed between lipid ratios and ECAS (see Fig. 1b).

Box-plots of the TC/HDL-C (see Fig. 2a), LDL-C/HDL$\mathrm{C}$ (see Fig. 2b), RC/HDL-C (see Fig. 2c), Non-HDL-C/ HDL-C (see Fig. 2d), Apo B/ HDL-C (see Fig. 2e), and Apo B/Apo A-I ratios (see Fig. 2f) were shown. Patients with ICAS had significantly higher LDL-C/HDL-C (see Fig. 2b), apo B/HDL-C (see Fig. 2e), and apo B/apo A-I ratios (see Fig. 2f) than those without ICAS in both sexes $(P<0.05)$. However, this difference was not observed for the TG/HDL-C (see Fig. 2a) and RC/HDL-C ratios (see Fig. 2c). A significant difference in the non-HDL-C/HDL$\mathrm{C}$ ratio was observed only in men (see Fig. 2d). Because the result was similar to that of the non-HDL-C/HDL-C ratio, the TC/HDL-C ratio was not presented (see Fig. 2).

Analysis of ROC curves revealed that the apo B/apo A-I ratio exhibited the highest AUC value (0.588) for ICAS among all of the lipid levels alone and the lipid ratios (see Table 2). However, the apo B/apo A-I ratio exhibited the highest AUC value only in men but not in women (men: 0.579; women: 0.613). In women, the apo $\mathrm{B} / \mathrm{HDL}-\mathrm{C}$ ratio demonstrated an optimal AUC value (0.617) for predicting ICAS risk. Overall, lipid ratios had higher AUC values than those for the lipid levels alone for the identification of ICAS. Additionally, the AUC in women was higher than that in men for all lipid ratios analyzed.

\section{Discussion}

This study revealed that the TC/HDL-C, RC/HDL-C, LDL-C/HDL-C, non-HDL-C/HDL-C, apo B/HDL-C, and apo B/apo A-I ratios were significantly correlated with ICAS. Moreover, ROC analyses revealed that lipid ratios were better than lipid levels alone for predicting ICAS. The apo B/apo A-I ratio had a higher predictive value than that of other variables. This is the first study to present these relationships in a Chinese population.

The correlation between the apo B/apo A-I ratio and ICAS was established in a Korean population by Park and colleagues. They demonstrated that a higher apo B/ apo A-I ratio could be a discriminator for ICAS rather than ECAS [9]. Sun et al. [11] found that apoB/AI ratio could be an independent factor for risk stratification of 
Table 1 Characteristics of the study participants according to gender

\begin{tabular}{|c|c|c|c|}
\hline Variables & Men $(n=441)$ & Women $(n=217)$ & $P$ Value \\
\hline Age, year & $64.6 \pm 11.2$ & $68.5 \pm 10.7$ & $<0.001$ \\
\hline Systolic blood pressure, $\mathrm{mmHg}$ & $152.8 \pm 23.8$ & $154.1 \pm 26.0$ & 0.522 \\
\hline Diastolic blood pressure, $\mathrm{mmHg}$ & $88.7 \pm 16.0$ & $84.7 \pm 14.6$ & 0.002 \\
\hline Hypertension, n (\%) & $311(70.5)$ & $165(76.0)$ & 0.137 \\
\hline Diabetes mellitus, n (\%) & $144(32.7)$ & $74(34.1)$ & 0.711 \\
\hline Current smoking, n (\%) & $251(56.9)$ & $4(1.8)$ & $<0.001$ \\
\hline Previous stroke, n (\%) & $131(29.7)$ & $56(25.8)$ & 0.297 \\
\hline Coronary heart disease, n (\%) & $51(11.6)$ & $29(13.4)$ & 0.507 \\
\hline Number of ICAS lesions & $0(0-1)$ & $0(0-1)$ & 0.771 \\
\hline NCAS & $238(54.0)$ & $128(59.0)$ & 0.223 \\
\hline Isolated ICAS & $115(26.1)$ & $67(30.9)$ & 0.196 \\
\hline Isolated ECAS & $38(8.6)$ & $8(3.7)$ & 0.020 \\
\hline ICAS and ECAS & $50(11.3)$ & $14(6.5)$ & 0.047 \\
\hline $\mathrm{TC}, \mathrm{mmol} / \mathrm{L}$ & $4.35 \pm 1.06$ & $4.67 \pm 1.10$ & $<0.001$ \\
\hline $\mathrm{TG}, \mathrm{mmol} / \mathrm{L}$ & $1.66 \pm 1.23$ & $1.62 \pm 1.01$ & 0.664 \\
\hline $\mathrm{HDL}-\mathrm{C}, \mathrm{mmol} / \mathrm{L}$ & $1.09(0.94-1.24)$ & $1.26(1.09-1.51)$ & $<0.001$ \\
\hline $\mathrm{LDL}-\mathrm{C}, \mathrm{mmol} / \mathrm{L}$ & $2.82 \pm 0.91$ & $2.97 \pm 0.99$ & 0.061 \\
\hline Non-HDL-C, mmol/L & $3.16(2.48-3.90)$ & $3.21(2.53-3.96)$ & 0.384 \\
\hline $\mathrm{RC}, \mathrm{mmol} / \mathrm{L}$ & $0.42 \pm 0.40$ & $0.37 \pm 0.35$ & 0.121 \\
\hline Apo A-I, g/L & $1.22 \pm 0.35$ & $1.40 \pm 0.27$ & $<0.001$ \\
\hline Apo B, g/L & $0.99 \pm 0.53$ & $0.96 \pm 0.29$ & 0.520 \\
\hline \multicolumn{4}{|l|}{ Lipid Ratio } \\
\hline $\mathrm{TC} / \mathrm{HDL}-\mathrm{C}$ & $4.12 \pm 1.37$ & $3.74 \pm 1.22$ & 0.001 \\
\hline $\mathrm{TG} / \mathrm{HDL}-\mathrm{C}$ & $1.71 \pm 1.77$ & $1.45 \pm 1.67$ & 0.069 \\
\hline LDL-C/HDL-C & $2.68 \pm 1.06$ & $2.39 \pm 0.94$ & 0.001 \\
\hline $\mathrm{RC} / \mathrm{HDL}-\mathrm{C}$ & $0.44 \pm 0.53$ & $0.35 \pm 0.55$ & 0.036 \\
\hline Non- HDL-C/HDL-C & $3.12 \pm 1.37$ & $2.73 \pm 1.22$ & 0.001 \\
\hline Apo B/HDL-C & $2.25(1.65-2.91)$ & $1.87(1.44-2.48)$ & $<0.001$ \\
\hline Apo B/apo A-I & $0.78(0.60-0.98)$ & $0.69(0.53-0.86)$ & $<0.001$ \\
\hline
\end{tabular}

ICAS intracranial atherosclerotic stenosis, ECAS extracranial atherosclerotic stenosis, NCAS no cerebral atherosclerotic stenosis, TC total cholesterol, TG triglycerides, LDL-C low-density lipoprotein cholesterol, HDL-C high-density lipoprotein cholesterol, $R C$ remnant cholesterol, Non-HDL-C non-high-density lipoprotein cholesterol, Apo $B$ apolipoprotein B, Apo A-I apolipoprotein A-I

Results are expressed as mean \pm standard deviation, median with interquartile range or $\mathrm{n}(\%)$

ICAS in both stroke patients and non-stroke controls, which is similar to the results of this study. Moreover, this ratio has also been shown to be superior to other ratios for the identification of coronary artery lesions in a Chinese population and coronary disease in a Swedish population [13, 21]. Thus, the apo B/apo A-I ratio is considered to be an excellent surrogate for the prediction of vascular disease risk. Nevertheless, few studies have focused on the apo B/HDL-C ratio. Maki and colleagues demonstrated that the apo B/apo A-I ratio can be a predictor of CIMT progression in vascular walls [22]. Biswas et al. [23] demonstrated that this ratio correlated well with the risk of coronary heart disease among Indian populations. Atherosclerosis is one of the main contributory indicator for coronary heart disease $[24,25]$. Hence, the apo B/HDL-C ratio has been proposed to be a marker of atherosclerosis.

This study also identified the important predictive abilities of the apo B/HDL-C and apo B/apo A-I ratios for ICAS rather than ECAS. This phenomenon could be explained by the greater effect on antioxidant enzymes in ICAS than in ECAS [26]. In an experimental study, dyslipidemic mice were characterized by increased oxidation of apoB in the blood and impaired HDLassociated antioxidative defense [27]. Moreover, apoA-I is considered a marker of antioxidant and anti- 


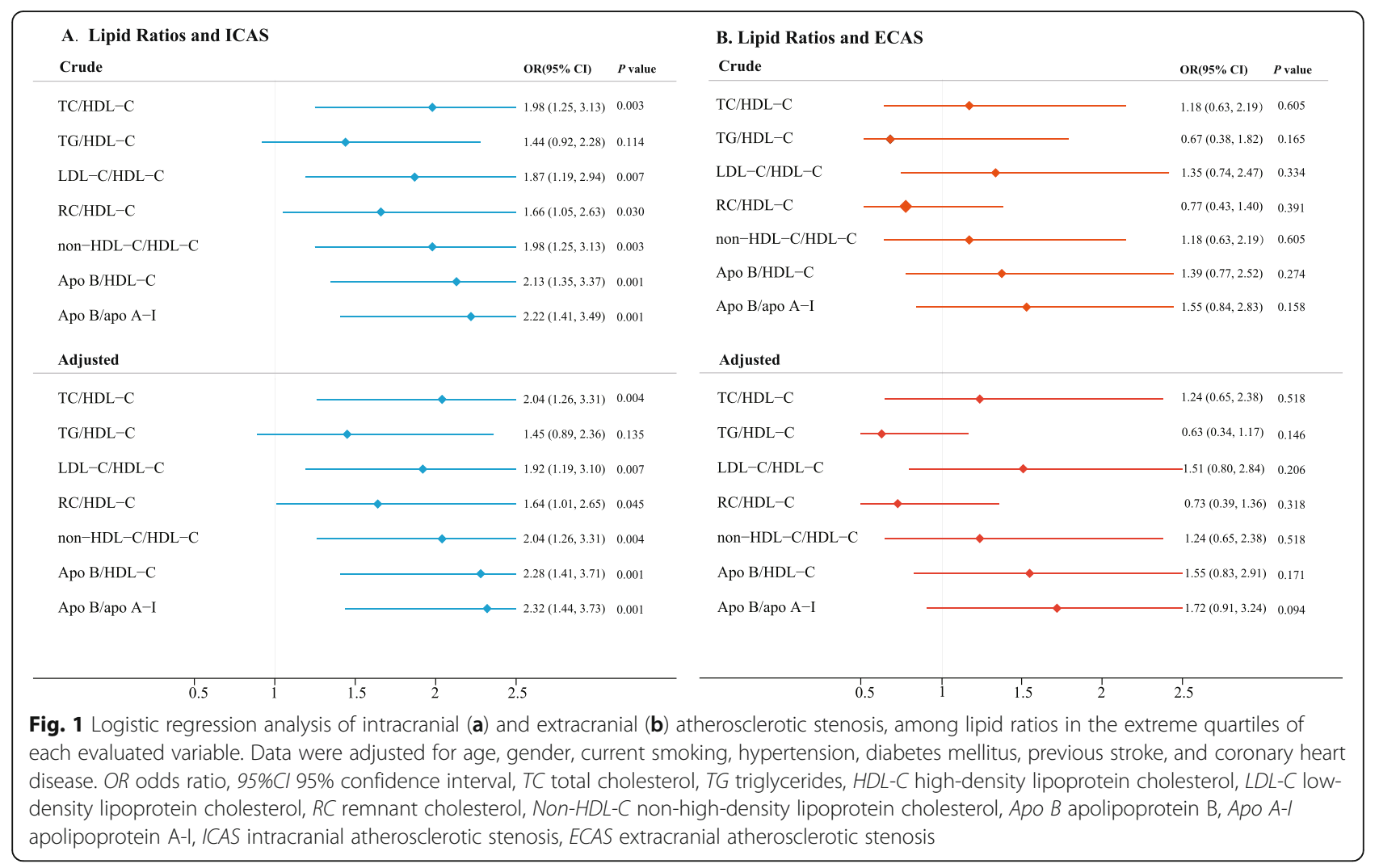

inflammatory properties [28], and is closely associated with the patients with pre-existing ischemic stroke [29]. Thus, this deficit in antioxidant protection might be the pathomechanism of ischemic stroke in ICAS in patients with high apoB/apoA-I ratios [29].

As the crucial lipoprotein in intermediate-density lipoprotein (IDL), very low-density lipoprotein (VLDL), as well as LDL and apo B, can reflect the potential atherogenic lipoprotein particles in lipid metabolism [30]. Conversely, apo A-I is the main component in HDL-C, which has anti-atherogenic and anti-inflammatory potential [31]. HDL-C can reverse cholesterol transport. Apo A-I and HDL-C have valuable antioxidant capacities. Therefore, the apo B/HDL-C and apo B/apo A-I ratios can more comprehensively reflect atherogenicity and lower antioxidant capacity.

Non-HDL-C integrates multiple types of cholesterol, including IDL, LDL, VLDL, and lipoprotein (a) and can be determined simply by calculation (TC level minus the HDL-C level) [17]. Because of the protective role of HDL-C against cardiovascular diseases, non-HDL-C has an atherogenic effect in the circulation. This association between non-HDL-C and ICAS has been confirmed in a Chinese population [6]. However, the diagnostic ability of the non-HDL-C/HDL-C ratio for ICAS has not been investigated. Moreover, the non-HDL-C/HDL-C ratio possesses good predictive ability for some diseases. This ratio is more useful than the apo B/apo A-I ratio for identifying the metabolic syndrome in a Korean population. In addition, a large retrospective study demonstrated a positive correlation between insulin resistance and C-reactive protein levels [32]. Among individuals with obesity and insulin resistance syndromes, lower HDL-C and higher non-HDL-C levels demonstrated associations with coronary heart disease in regression models [33]. Moreover, non-HDL-C/HDL-C ratio is a strong indicator for predicting carotid atherosclerotic plaque in middle-aged postmenopausal women [34]. Besides, for the prediction of risk of cardiovascular diseases, this ratio is similar to the apo B/apo A-I ratio for DM patients [35]. Compared with traditional lipid variables, this ratio is more suitable for the estimation of arterial stiffness in a Chinese population [36]. In this study, the non-HDL-C/HDL-C ratio was associated with a two-fold risk of ICAS. Therefore, control of this ratio may be important for ICAS risk. The results of the TC/ HDL-C ratio were similar to those of the non-HDL-C/ HDL-C ratio.

RC comprises the TG-rich lipoproteins IDL, VLDL, and chylomicrons [18]. RC is regarded as a causal indicator of cardiovascular diseases [37, 38]. The diagnostic values of $\mathrm{RC}$ and the $\mathrm{RC} / \mathrm{HDL}-\mathrm{C}$ ratio have been 

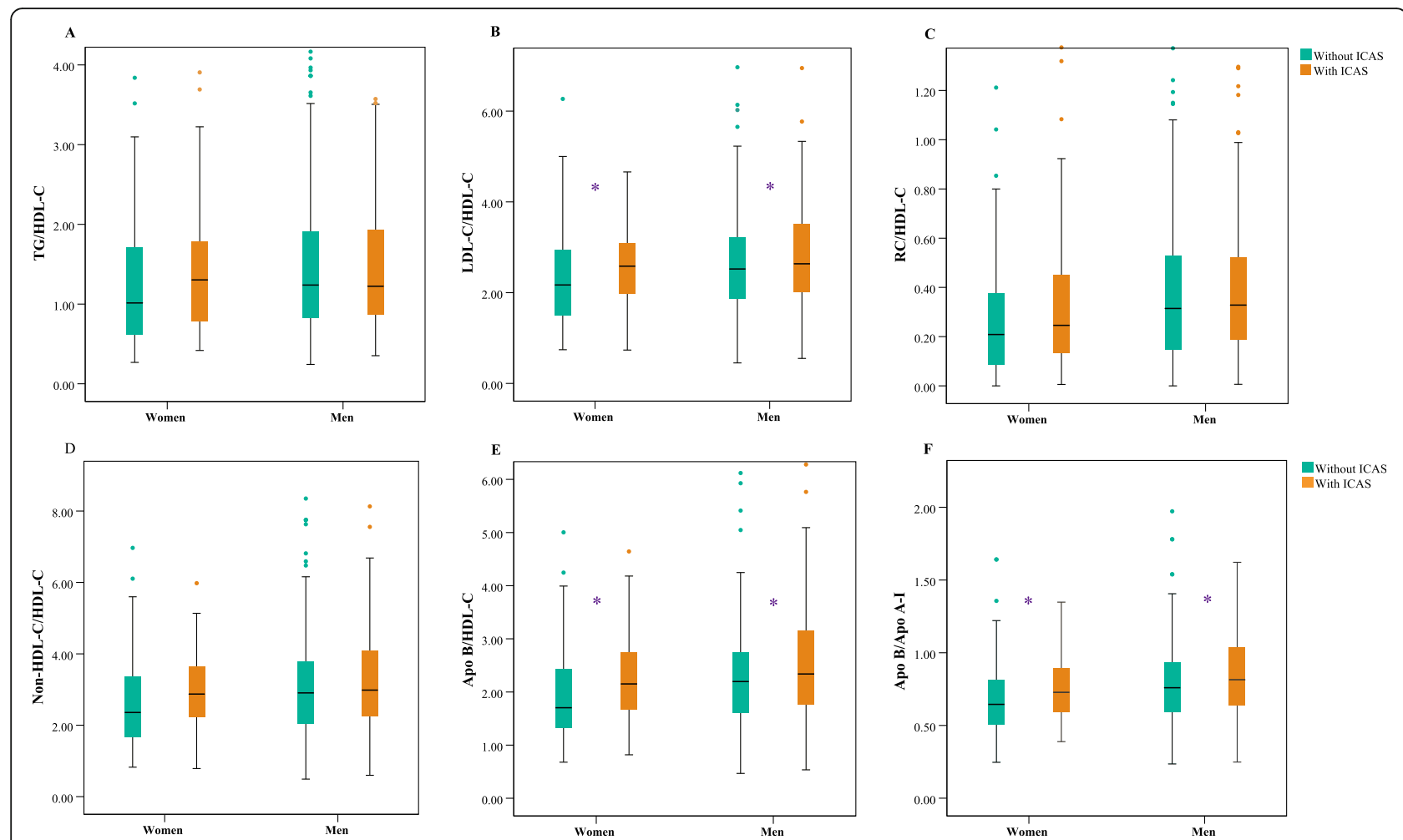

Fig. 2 Box-plots of lipid ratios values and their association with ICAS in men and women. The total cholesterol (TC)/high-density lipoprotein cholesterol (HDL-C) (a), low-density lipoprotein cholesterol (LDL-C)/HDL-C (b), remnant cholesterol (RC)/HDL-C (c), non-high-density lipoprotein cholesterol (Non-HDL-C)/HDL-C (d), apolipoprotein B (Apo B)/HDL-C (e), and Apo B/apolipoprotein A-I (Apo A-I) (f) ratios are shown. TG triglycerides, ICAS intracranial atherosclerotic stenosis. *Significant difference at $P<0.05$

identified simultaneously in Chinese patients with periprocedural myocardial injury [39]. The RC/HDL-C ratio appears to be a useful tool for assessing the risk of ICAS. Compared with other important lipid ratios, only the $\mathrm{RC} / \mathrm{HDL}-\mathrm{C}$ ratio showed a slight association with ICAS in this study. The exact mechanism of action is not clear, but low-grade inflammation caused by RC could be one reason for this association. Subsequently, RC could enter vascular walls by infiltrating the endothelial barrier, and lead to the formation of foam cells by upregulating the expression of scavenger receptors [40].

\section{Study strengths and limitations}

This study had several strengths. First, this study not only verified the data of other studies, but it also clarified the specific diagnostic value of lipid ratios upon ICAS. This study found that lipid ratios were better than routinely used lipid concentrations for predicting ICAS. Second, ROC curves indicated that the diagnostic ability of the apo B/apo A-I ratio surpassed those of all other ratios tested. This ratio was determined to be the best marker for ICAS risk. The lipid ratios indicated the balance between anti-atherogenic and pro-atherogenic mechanisms. The combined effects of lipid ratios could be more valuable than lipid levels alone. Third, this study suggested that lipid ratios had better predictive values than those of lipid levels alone for identifying ICAS risk. Finally, sex-based differences were examined regarding the predictive accuracy of lipid profiles. The apo B/apo A-I ratio had the highest AUC value for men, and the apo $\mathrm{B} / \mathrm{HDL}-\mathrm{C}$ ratio displayed the highest $\mathrm{AUC}$ value for women. One interpretation is that estrogen may exert an effect on lipid profiles, but the exact cause is not known, and further investigation is needed.

This study had five main limitations. First, data from a relatively small hospital-based population cannot be generalized to larger populations. Second, given the crosssectional design and a relatively small cohort of patients with symptomatic ischemic stroke, a causal relationship between lipid ratios and ICAS could not be accurately ascertained. Third, this study focused on a Chinese population, therefore, the conclusions cannot be extrapolated to different ethnic groups. Fourth, cerebral atherosclerotic stenosis was evaluated using DSA and CTA. These methods have a high degree of accuracy for evaluating the severity of stenosis but are less accurate for cerebral atherosclerotic stenosis. Fifth, the absence of data such as insulin resistance, inflammatory indicators, menopausal status, the use of hypolipidemic drugs or anti-hypertensives drugs, apo CIII, apo-E, lipoprotein(a), 
Table 2 Comparison of AUC for each evaluated variable in predicting ICAS

\begin{tabular}{|c|c|c|c|}
\hline & Total & Men & Women \\
\hline & $\mathrm{AUC}(95 \% \mathrm{Cl})$ & $\mathrm{AUC}(95 \% \mathrm{Cl})$ & $\mathrm{AUC}(95 \% \mathrm{Cl})$ \\
\hline \multicolumn{4}{|l|}{ Lipid measures } \\
\hline TC & $0.543(0.498-0.589)$ & $0.537(0.481-0.592)$ & $0.562(0.482-0.642)$ \\
\hline TG & $0.525(0.480-0.570)$ & $0.499(0.444-0.554)$ & $0.576(0.500-0.652)$ \\
\hline $\mathrm{HDL}-\mathrm{C}$ & $0.462(0.417-0.507)$ & $0.480(0.426-0.535)$ & $0.435(0.356-0.514)$ \\
\hline LDL-C & $0.555(0.510-0.601)$ & $0.547(0.491-0.603)$ & $0.573(0.493-0.653)$ \\
\hline Non-HDL-C & $0.559(0.513-0.604)$ & $0.543(0.488-0.599)$ & $0.590(0.511-0.669)$ \\
\hline $\mathrm{RC}$ & $0.538(0.493-0.583)$ & $0.529(0.475-0.584)$ & $0.558(0.481-0.635)$ \\
\hline Apo A-I & $0.456(0.411-0.501)$ & $0.465(0.410-0.520)$ & $0.424(0.345-0.504)$ \\
\hline Apo B & $0.576(0.531-0.622)$ & $0.561(0.505-0.617)$ & $0.605(0.527-0.683)$ \\
\hline \multicolumn{4}{|l|}{ Lipid ratios } \\
\hline $\mathrm{TC} / \mathrm{HDL}-\mathrm{C}$ & $0.566(0.521-0.611)$ & $0.546(0.491-0.601) *$ & $0.608(0.531-0.684)$ \\
\hline TG/HDL-C & $0.535(0.490-0.579)$ * & $0.511(0.456-0.566) *$ & $0.580(0.503-0.657)$ \\
\hline LDL-C/HDL-C & $0.570(0.525-0.615)$ & $0.555(0.499-0.611)$ & $0.603(0.526-0.680)$ \\
\hline $\mathrm{RC} / \mathrm{HDL}-\mathrm{C}$ & $0.544(0.500-0.589)$ & $0.530(0.475-0.584)$ & $0.575(0.498-0.652)$ \\
\hline Non- HDL-C/HDL-C & $0.566(0.521-0.611)$ & $0.546(0.491-0.601) *$ & $0.608(0.531-0.684)$ \\
\hline Apo B/ HDL-C & $0.578(0.533-0.623)$ & $0.562(0.506-0.617)$ & $0.617(0.541-0.693)$ \\
\hline Apo B/apo A-I & $0.588(0.543-0.633)$ & $0.579(0.523-0.634)$ & $0.613(0.536-0.690)$ \\
\hline
\end{tabular}

$T C$ total cholesterol, TG triglycerides, LDL-C low-density lipoprotein cholesterol, HDL-C high-density lipoprotein cholesterol, $R C$ remnant cholesterol, Non-HDL-C non-high-density lipoprotein cholesterol, Apo B apolipoprotein B, Apo A-I apolipoprotein A-I, AUC area under the curve; $95 \% \mathrm{Cl} 95 \%$ confidence interval, ICAS intracranial atherosclerotic stenosis. * $P<0.05$, AUC were compared with apo B/apo A-I ratio in lipid ratios

and dietary habits may have a potential influence on outcomes.

\section{Conclusion}

The TC/HDL-C, LDL-C/HDL-C, RC/HDL-C, non$\mathrm{HDL}-\mathrm{C} / \mathrm{HDL}-\mathrm{C}$, and apo $\mathrm{B} / \mathrm{HDL}-\mathrm{C}$ ratios were significantly related to ICAS risk. Compared with the other variables tested, the apo B/apo A-I ratio appeared to be a better discriminator for identifying ICAS risk. Lipid ratios could be potential indicators for risk stratification of ICAS, and provide guidance in lipid-lowering therapy in patients with symptomatic ischemic stroke.

\section{Supplementary information}

Supplementary information accompanies this paper at https://doi.org/10. 1186/s12944-020-01336-1.

\section{Additional file 1.}

\section{Abbreviations}

Apo A-I: Apolipoprotein A-I; Apo B: Apolipoprotein B; ICAS: Intracranial atherosclerotic stenosis; ECAS: Extracranial atherosclerotic stenosis; Non-HDLC: Non-high-density lipoprotein cholesterol; RC: Remnant cholesterol; TC: Total cholesterol; TG: Triglycerides; LDL-C: Low-density lipoprotein cholesterol; HDL-C: High-density lipoprotein cholesterol; SE: Indicates standard error; $\beta$ : Regression coefficients; OR: Odds ratio; 95\%Cl: 95\% confidence interval; AUC: Area under the curve; DSA: Digital subtraction angiography; CTA: Computed tomography angiography; CIMT: Carotid intima-media thickness; DM: Diabetes mellitus; IDL: Intermediate-density lipoprotein; VLDL: Very low-density lipoprotein; NCAS: No cerebral atherosclerotic stenosis

\section{Acknowledgements \\ None}

\section{Authors' contributions}

Wen-Song Yang, Rui Li, Yi-Qing Shen, Qi Li, Guo-En Yao, and Peng Xie conceived and designed the study. Xing-Chen Wang, Qing-Jun Liu, and Hai-Yang Wang coordinated the study. Wen-Song Yang, Rui Li, Yi-Qing Shen, Xing-Chen Wang, Qing-Jun Liu, and Hai-Yang Wang did the acquisition, analysis, or interpretation of data. Qi Li, Guo-En Yao, and Peng Xie oversaw subjects' recruitment and monitored the gathering of clinical data. Wen-Song Yang, Rui $\mathrm{Li}$, and Yi-Qing Shen conducted the statistical analysis and prepared the paper. Qi Li and Peng Xie obtained fundings. Qi Li, Guo-En Yao, and Peng Xie were responsible for the administrative, technical, or material support. The author(s) read and approved the final manuscript.

Funding

The Special Project on Natural Chronic Non-infectious Diseases (2016YFC1307200) and the National Key Research and Development Program of China (2017YFA0505700).

Availability of data and materials

The datasets used and/or analyzed during the current study are available from the corresponding author on reasonable request.

\section{Ethics approval and consent to participate}

The study was approved by the ethics committee of the First Affiliated Hospital of Chongqing Medical University. (No. 2007-002).

Consent for publication

Written informed consent was obtained from all participants. 


\section{Competing interests}

None of the authors reports any disclosures.

\section{Author details}

'Department of Neurology, The First Affiliated Hospital of Chongqing Medical University, Chongqing 400016, China. ${ }^{2}$ NHC Key Laboratory of Diagnosis and Treatment on Brain Functional Diseases, The First Affiliated Hospital of Chongqing Medical University, Chongqing 400016, China. ${ }^{3}$ Division of Life Sciences and Medicine, Department of Neurology, The First Affiliated Hospital of USTC, University of Science and Technology of China, Hefei, Anhui 230001, P.R. China. ${ }^{4}$ Department of Psychiatry, The First People's Hospital of Yunnan Province, Kunming 650032, Yunnan, China. ${ }^{5}$ Department of Neurology, Yongchuan Hospital of Chongqing Medical University, Chongqing 402160, China. ${ }^{6}$ Department of Neurology, First Affiliated Hospital, PLA General Hospital, Beijing 100048, China.

\section{Received: 23 March 2020 Accepted: 24 June 2020}

\section{Published online: 04 July 2020}

\section{References}

1. Qureshi Al, Caplan LR. Intracranial atherosclerosis. Lancet. 2014;383:984-98. https://doi.org/10.1016/S0140-6736(13)61088-0.

2. Holmstedt CA, Turan TN, Chimowitz MI. Atherosclerotic intracranial arterial stenosis: risk factors, diagnosis, and treatment. The Lancet Neurology. 2013; 12:1106-14. https://doi.org/10.1016/S1474-4422(13)70195-9.

3. Kim JS, Bonovich D. Research on intracranial atherosclerosis from the east and west: why are the results different? J Stroke. 2014;16:105-13. https://doi. org/10.5853/jos.2014.16.3.105.

4. Ritz K, Denswil NP, Stam OCG, van Lieshout JJ, Daemen MJAP. Cause and mechanisms of intracranial atherosclerosis. Circulation. 2014;130:1407-14. https://doi.org/10.1161/CIRCULATIONAHA.114.011147.

5. Qian Y, Pu Y, Liu L, Wang DZ, Zhao X, Wang C, et al. Low HDL-C level is associated with the development of intracranial artery stenosis: analysis from the Chinese IntraCranial AtheroSclerosis (CICAS) study. PLoS One. 2013; 8:e64395. https://doi.org/10.1371/journal.pone.0064395.

6. Wu J, Zhang Q, Yang H, Gao X, Zhou Y, Wang A, et al. Association between non-high-density-lipoprotein-cholesterol levels and the prevalence of asymptomatic intracranial arterial stenosis. PLoS One. 2013;8:e65229. https://doi.org/10.1371/journal.pone.0065229.

7. Shen Y, Wang J, Wu J, Qu W, Wang C, Gao X, et al. Elevated plasma total cholesterol level is associated with the risk of asymptomatic intracranial arterial stenosis. PLoS One. 2014;9:e101232. https://doi.org/10.1371/journal. pone.0101232.

8. Kim Y-S, Hong J-W, Jung W-S, Park S-U, Park J-M, Cho S-I, et al. Gender differences in risk factors for intracranial cerebral atherosclerosis among asymptomatic subjects. Gend Med. 2011;8:14-22. https://doi.org/10.1016/j. genm.2011.01.001.

9. Park J-H, Hong K-S, Lee E-J, Lee J, Kim D-E. High levels of apolipoprotein B/ Al ratio are associated with intracranial atherosclerotic stenosis. Stroke. 2011; 42:3040-6. https://doi.org/10.1161/STROKEAHA.111.620104.

10. Kim D-E, Kim J-Y, Jeong S-W, Cho Y-J, Park J-M, Lee J-H, et al. Association between changes in lipid profiles and progression of symptomatic intracranial atherosclerotic stenosis: a prospective multicenter study. Stroke. 2012:43:1824-30. https://doi.org/10.1161/STROKEAHA.112.653659.

11. Sun Y, Hou X-H, Wang D-D, Ma Y-H, Tan C-C, Sun F-R, et al. Apolipoprotein $\mathrm{B} / \mathrm{Al}$ ratio as an independent risk factor for intracranial atherosclerotic stenosis. Aging (Albany NY). 2019;11:6851-62. https://doi.org/10.18632/ aging.102216.

12. Enomoto M, Adachi H, Hirai Y, Fukami A, Satoh A, Otsuka M, et al. LDL-C/ HDL-C ratio predicts carotid intima-media thickness progression better than HDL-C or LDL-C alone. J Lipids. 2011;2011:549137. https://doi.org/10.1155/ 2011/549137.

13. Hong L-F, Yan X-N, Fan Y, Wu Q, Luo S-H, Yang B, Li J-J. Is the ratio of apoB/apoA-1 the best predictor for the severity of coronary artery lesions in Chinese diabetics with stable angina pectoris? An assessment based on Gensini scores. J Geriatr Cardiol. 2015;12:402-9. https://doi.org/10.11909/j. issn.1671-5411.2015.04.012

14. Byun AR, Lee SW, Lee HS, Shim KW. What is the most appropriate lipid profile ratio predictor for insulin resistance in each sex? A cross-sectional study in Korean populations (the fifth Korea National Health and nutrition examination survey). Diabetol Metab Syndr. 2015;7:59. https://doi.org/10. 1186/s13098-015-0051-2.

15. Abbasian M, Delvarianzadeh M, Ebrahimi $H$, Khosravi F. Lipid ratio as a suitable tool to identify individuals with MetS risk: a case- control study. Diabetes Metab Syndr. 2017;11(Suppl 1):S15-9. https://doi.org/10.1016/j.dsx. 2016.08.011.

16. Zhai F-F, Yan S, Li M-L, Han F, Wang Q, Zhou L-X, et al. Intracranial arterial Dolichoectasia and stenosis: risk factors and relation to cerebral small vessel disease. Stroke. 2018:49:1135-40. https://doi.org/10.1161/STROKEAHA.117. 020130.

17. Expert Panel on Detection, Evaluation, and Treatment of High Blood Cholesterol in Adults. Executive Summary of The Third Report of The National Cholesterol Education Program (NCEP) Expert Panel on Detection, Evaluation, And Treatment of High Blood Cholesterol In Adults (Adult Treatment Panel III). JAMA. 2001;285:2486-97. https://doi.org/10.1001/jama. 285.19.2486

18. Nordestgaard BG, Varbo A. Triglycerides and cardiovascular disease. Lancet. 2014;384:626-35. https://doi.org/10.1016/S0140-6736(14)61177-6.

19. Samuels OB, Joseph GJ, Lynn MJ, Smith HA, Chimowitz MI. A standardized method for measuring intracranial arterial stenosis. AJNR Am J Neuroradiol. 2000;21:643-6.

20. Barnett HJM, Taylor DW, Haynes RB, Sackett DL, Peerless SJ, Ferguson GG, et al. Beneficial effect of carotid endarterectomy in symptomatic patients with high-grade carotid stenosis. N Engl J Med. 1991;325:445-53. https://doi. org/10.1056/NEJM199108153250701.

21. Walldius G, Jungner I, Holme I, Aastveit AH, Kolar W, Steiner E. High apolipoprotein B, low apolipoprotein A-I, and improvement in the prediction of fatal myocardial infarction (AMORIS study): a prospective study. Lancet. 2001;358:2026-33. https://doi.org/10.1016/S01406736(01)07098-2.

22. Maki KC, Davidson MH, Dicklin MR, Bell M, Witchger M, Feinstein SB. Predictors of anterior and posterior wall carotid intima media thickness progression in men and women at moderate risk of coronary heart disease. J Clin Lipidol. 2011;5:141-51. https://doi.org/10.1016/j.jacl.2011.02.003.

23. Biswas S, Ghoshal PK, Mandal SC, Mandal N. Association of low-density lipoprotein particle size and ratio of different lipoproteins and apolipoproteins with coronary heart disease. J Cardiol. 2008:52:118-26. https://doi.org/10.1016/j.jjcc.2008.06.005.

24. Herrington DM, Mao C, Parker SJ, Fu Z, Yu G, Chen L, et al. Proteomic architecture of human coronary and aortic atherosclerosis. Circulation. 2018; 137:2741-56. https://doi.org/10.1161/CIRCULATIONAHA.118.034365.

25. Simon A, Chironi G, Levenson J. Comparative performance of subclinical atherosclerosis tests in predicting coronary heart disease in asymptomatic individuals. Eur Heart J. 2007;28:2967-71. https://doi.org/10.1093/eurheartj/ ehm487.

26. D'Armiento FP, Bianchi A, de Nigris F, Capuzzi DM, D'Armiento MR, Crimi G, et al. Age-related effects on atherogenesis and scavenger enzymes of intracranial and extracranial arteries in men without classic risk factors for atherosclerosis. Stroke. 2001;32:2472-9.

27. Mertens A, Verhamme P, Bielicki JK, Phillips MC, Quarck R, Verreth W, et al. Increased low-density lipoprotein oxidation and impaired high-density lipoprotein antioxidant defense are associated with increased macrophage homing and atherosclerosis in dyslipidemic obese mice: LCAT gene transfer decreases atherosclerosis. Circulation. 2003;107:1640-6. https://doi.org/10. 1161/01.CIR.0000056523.08033.9F.

28. Bielicki JK, Oda MN. Apolipoprotein A-I(Milano) and apolipoprotein A-I(Paris) exhibit an antioxidant activity distinct from that of wild-type apolipoprotein A-I. Biochemistry. 2002;41:2089-96. https://doi.org/10.1021/bi011716p.

29. Park J-H, Hong K-S, Lee J, Kim Y-J, Song P. Deep subcortical infarct burden in relation to apolipoprotein $\mathrm{B} / \mathrm{Al}$ ratio in patients with intracranial atherosclerotic stenosis. Eur J Neurol. 2013;20:671-80. https://doi.org/10. 1111/ene.12021.

30. Marcovina S, Packard CJ. Measurement and meaning of apolipoprotein Al and apolipoprotein B plasma levels. J Intern Med. 2006;259:437-46. https://doi.org/10.1111/j.1365-2796.2006.01648.x.

31. Zhu X, Parks JS. New roles of HDL in inflammation and hematopoiesis. Annu Rev Nutr. 2012. https://doi.org/10.1146/annurev-nutr-071811-150709.

32. Kim SW, Jee JH, Kim HJ, Jin S-M, Suh S, Bae JC, et al. Non-HDL-cholesterol/ $\mathrm{HDL}$-cholesterol is a better predictor of metabolic syndrome and insulin resistance than apolipoprotein B/apolipoprotein A1. Int J Cardiol. 2013;168: 2678-83. https://doi.org/10.1016/j.ijcard.2013.03.027. 
33. Ndumele CE, Matsushita K, Astor B, Virani SS, Mora S, Williams EK, et al. Apolipoproteins do not add prognostic information beyond lipoprotein cholesterol measures among individuals with obesity and insulin resistance syndromes: the ARIC study. Eur J Prev Cardiol. 2014;21:866-75. https://doi. org/10.1177/2047487312465523.

34. Masson W, Epstein T, Huerín M, Lobo M, Molinero G, Siniawski D. Association between non-HDL-C/HDL-C ratio and carotid atherosclerosis in postmenopausal middle-aged women. Climacteric. 2019;22:518-22. https://doi.org/10.1080/13697137.2019.1631787.

35. Taskinen M-R, Barter PJ, Ehnholm C, Sullivan DR, Mann K, Simes J, et al. Ability of traditional lipid ratios and apolipoprotein ratios to predict cardiovascular risk in people with type 2 diabetes. Diabetologia. 2010;53: 1846-55. https://doi.org/10.1007/s00125-010-1806-9.

36. Zhao W, Gong W, Wu N, Li Y, Ye K, Lu B, et al. Association of lipid profiles and the ratios with arterial stiffness in middle-aged and elderly Chinese. Lipids Health Dis. 2014;13:37. https://doi.org/10.1186/1476-511X-13-37.

37. Varbo A, Benn M, Tybjærg-Hansen A, Jørgensen AB, Frikke-Schmidt R, Nordestgaard BG. Remnant cholesterol as a causal risk factor for ischemic heart disease. J Am Coll Cardiol. 2013;61:427-36. https://doi.org/10.1016/j. jacc.2012.08.1026.

38. Varbo A, Benn M, Nordestgaard BG. Remnant cholesterol as a cause of ischemic heart disease: evidence, definition, measurement, atherogenicity, high risk patients, and present and future treatment. Pharmacol Ther. 2014 141:358-67. https://doi.org/10.1016/j.pharmthera.2013.11.008.

39. Zeng R-X, Li S, Zhang M-Z, Li X-L, Zhu C-G, Guo Y-L, et al. Remnant cholesterol predicts periprocedural myocardial injury following percutaneous coronary intervention in poorly-controlled type 2 diabetes. J Cardiol. 2017;70:113-20. https://doi.org/10.1016/j.jjcc.2016.12.010.

40. Varbo A, Benn M, Tybjærg-Hansen A, Nordestgaard BG. Elevated remnant cholesterol causes both low-grade inflammation and ischemic heart disease, whereas elevated low-density lipoprotein cholesterol causes ischemic heart disease without inflammation. Circulation. 2013;128:1298309. https://doi.org/10.1161/CIRCULATIONAHA.113.003008.

\section{Publisher's Note}

Springer Nature remains neutral with regard to jurisdictional claims in published maps and institutional affiliations.

Ready to submit your research? Choose BMC and benefit from:

- fast, convenient online submission

- thorough peer review by experienced researchers in your field

- rapid publication on acceptance

- support for research data, including large and complex data types

- gold Open Access which fosters wider collaboration and increased citations

- maximum visibility for your research: over $100 \mathrm{M}$ website views per year

At $\mathrm{BMC}$, research is always in progress.

Learn more biomedcentral.com/submissions 